\title{
Multivariable Proportional-Integral-Plus (PIP) control of the ALSTOM nonlinear gasifier simulation
}

\author{
C.J. Taylor and E.M. Shaban \\ Engineering Department, Lancaster University, Lancaster, LA1 4YR, UK
}

This paper is a postprint of a paper submitted to and accepted for publication in IEE Proceedings Control Theory and Applications and is subject to Institution of Engineering and Technology Copyright. The copy of record is available at IET Digital Library. [IEE Proceedings Control Theory and Applications 153, 3, 277-285, 2006] 


\begin{abstract}
Multivariable Proportional-Integral-Plus (PIP) control methods are applied to the nonlinear ALSTOM Benchmark Challenge II. The approach utilises a data-based combined model reduction and linearisation step, which plays an essential role in satisfying the design specifications. The discrete-time transfer function models obtained in this manner, are represented in a NonMinimum State Space (NMSS) form suitable for PIP control system design. Here, full state variable feedback control can be implemented directly from the measured input and output signals of the controlled process, without resort to the design and implementation of a deterministic state reconstructor or a stochastic Kalman filter. Furthermore, the non-minimal formulation provides more design freedom than the equivalent minimal case, a characteristic that proves particularly useful in tuning the algorithm to meet the Benchmark specifications. The latter requirements are comfortably met for all three operating conditions by using a straightforward to implement, fixed gain, linear PIP algorithm.
\end{abstract}

\title{
Keywords
}

ALSTOM Benchmark Challenge; identification; control system design; model-based control; proportional-integral-plus 


\section{Introduction}

This paper applies multivariable Proportional-Integral-Plus (PIP) control to the nonlinear ALSTOM Benchmark Challenge II. The PIP controller can be interpreted as a logical extension of conventional PI/PID algorithms, but with inherent model-based predictive control action $[1,2]$. Here, multivariable Non-Minimal State Space (NMSS) models are formulated so that full state variable feedback control can be implemented directly from the measured input and output signals of the controlled process, without resorting to the design of a deterministic state reconstructor (observer) or a stochastic Kalman filter.

Over the last few years, such NMSS/PIP control systems have been successfully employed in a range of practical and simulation studies [e.g. 3, 4, 5], including the 1998 Gasifier Challenge [6]. The latter research was based on the same pilot integrated plant for an air blown gasification cycle, as that utilised in the present study [7, 8]. However, the 1998 challenge considered a high order linearised version of the gasifier simulation. Here, a discretetime PIP algorithm satisfied all of the performance requirements for both the 100\% and 50\% load operating conditions. This solution involved a very simple design procedure, with just one weighting term used to straightforwardly tune the closed loop response [6]. 
The present paper follows on from this earlier research by now applying the NMSS/PIP methodology to the nonlinear simulation, requiring an appropriate linearisation step. In fact, the research utilises the Simplified Refined Instrumental Variable (SRIV) algorithm $[9,10]$ to estimate multiinput, single-output, linear Transfer Function (TF) models for the system, i.e. a combined model reduction and linearisation exercise.

For clarity, the notation used throughout the paper is reviewed below. The model includes 5 actuators, all flow rates with units of $\mathrm{kg} / \mathrm{s}$ : char extraction (WCHR), air mass (WAIR), coal (WCOL), steam mass (WSTM) and limestone mass (WLS). However, the specifications require that WLS is always set to $10 \%$ of the value of WCOL, effectively leaving 4 controllable inputs to decouple the 4 outputs. These outputs include: fuel gas calorific value (CVGAS, MJ/kg), bed mass (MASS, tons), fuel gas pressure (PGAS, bars) and fuel gas temperature (TGAS, K). The units listed here are used consistently in all the figures below. Full details and performance tests are described in the introductory paper to this special issue [8].

Section 2 of the paper briefly reviews the multivariable NMSS/PIP approach to control system design. Appropriate linear transfer function models are identified for the gasifier system in Section 3. This is followed in Section 4 by the standard benchmark performance tests while, finally, the conclusions are presented in Section 5 . 


\section{NMSS/PIP Control}

Multivariable PIP control can be applied to systems represented by either discrete-time, backward shift [11] and delta $(\delta)[12]$ operator, or continuoustime (derivative operator) models. However, backward shift methods are employed for the research described below since they are so straightforward, yet are found to yield very good control of the 'stiff' gasifier system, which includes an array of fast and very slow dynamic modes. In this case, consider the following $p$-input, $p$-output, left Matrix Fraction Description,

$$
\begin{aligned}
\mathbf{y}(k) & =\left[\mathbf{A}\left(z^{-1}\right)\right]^{-1} \mathbf{B}\left(z^{-1}\right) \mathbf{u}(k) \\
\mathbf{y}(k) & =\left[y_{1}(k), y_{2}(k), \ldots, y_{p}(k)\right]^{T} \\
\mathbf{u}(k) & =\left[u_{1}(k), u_{2}(k), \ldots, u_{p}(k)\right]^{T} \\
\mathbf{A}\left(z^{-1}\right) & =\mathbf{I}+\mathbf{A}_{1} z^{-1}+\ldots+\mathbf{A}_{n} z^{-n} \\
\mathbf{B}\left(z^{-1}\right) & =\mathbf{B}_{1} z^{-1}+\ldots+\mathbf{B}_{m} z^{-m}
\end{aligned}
$$

Here, $\mathbf{y}(k)$ and $\mathbf{u}(k)$ are vectors of system outputs and control inputs respectively, $\mathbf{A}_{i}(i=1,2, \ldots, n)$ and $\mathbf{B}_{i}(i=1,2, \ldots, m)$ are $p$ by $p$ matrices of model coefficients, while $z^{-1}$ is the backward shift operator, i.e. $z^{-i} y(k)=y(k-i)$. For the gasifier simulation with $p=4, \mathbf{y}(k)$ consists of the CVGAS, MASS, PGAS and TGAS variables, while $\mathbf{u}(k)$ is similarly formed from WCHR, WAIR, WCOL and WSTM. 
Equation (1) is formulated from linear TF models identified for each input-output pathway of the multivariable system, as discussed in Section 3. The NMSS representation is subsequently defined as follows,

$$
\begin{gathered}
\mathbf{x}(k)=\mathbf{F} \mathbf{x}(k-1)+\mathbf{G u}(k-1)+\mathbf{D} \mathbf{y}_{d}(k) \\
\mathbf{y}(k)=\mathbf{H} \mathbf{x}(k)
\end{gathered}
$$

Here, the non-minimal state vector is given by,

$$
\begin{aligned}
\mathbf{x}(k)=[\mathbf{y}(k), \mathbf{y}(k-1), \cdots, \mathbf{y}(k-n+1), \\
\mathbf{u}(k-1), \cdots, \mathbf{u}(k-m+1), \mathbf{z}(k)]^{T}
\end{aligned}
$$

where,

$$
\mathbf{z}(k)=\mathbf{z}(k-1)+\left[\mathbf{y}_{d}(k)-\mathbf{y}(k)\right]
$$

The latter is called the integral-of-error vector, in which $\mathbf{y}_{d}(k)$ is the reference or command input vector, each element being associated with the relevant system output. Inherent type 1 servomechanism performance is introduced by means of the state variables in $\mathbf{z}(k)$. If the closed-loop system is stable, then this ensures that steady-state decoupling is inherent in the basic design.

The state transition $\mathbf{F}$, input $\mathbf{G}$, command $\mathbf{D}$ and observation $\mathbf{H}$ matrices of the NMSS system are subsequently defined below, 


$$
\begin{aligned}
& \mathbf{F}=\left[\begin{array}{ccccccccccc}
-\mathbf{A}_{1} & -\mathbf{A}_{2} & \cdots & -\mathbf{A}_{n-1} & -\mathbf{A}_{n} & \mathbf{B}_{2} & \mathbf{B}_{3} & \cdots & \mathbf{B}_{m-1} & \mathbf{B}_{m} & \mathbf{0} \\
\mathbf{I}_{p} & \mathbf{0} & \cdots & \mathbf{0} & \mathbf{0} & \mathbf{0} & \mathbf{0} & \cdots & \mathbf{0} & \mathbf{0} & \mathbf{0} \\
\mathbf{0} & \mathbf{I}_{p} & \cdots & \mathbf{0} & \mathbf{0} & \mathbf{0} & \mathbf{0} & \cdots & \mathbf{0} & & \mathbf{0} \\
\vdots & \vdots & \ddots & \vdots & \vdots & \vdots & \vdots & \ddots & \vdots & \vdots & \vdots \\
\mathbf{0} & \mathbf{0} & \cdots & \mathbf{I}_{p} & \mathbf{0} & \mathbf{0} & \mathbf{0} & \cdots & \mathbf{0} & \mathbf{0} & \mathbf{0} \\
\mathbf{0} & \mathbf{0} & \cdots & \mathbf{0} & \mathbf{0} & \mathbf{0} & \mathbf{0} & \cdots & \mathbf{0} & \mathbf{0} & \mathbf{0} \\
\mathbf{0} & \mathbf{0} & \cdots & \mathbf{0} & \mathbf{0} & \mathbf{I}_{p} & \mathbf{0} & \cdots & \mathbf{0} & \mathbf{0} & \mathbf{0} \\
\mathbf{0} & \mathbf{0} & \cdots & \mathbf{0} & \mathbf{0} & \mathbf{0} & \mathbf{I}_{p} & \cdots & \mathbf{0} & \mathbf{0} & \mathbf{0} \\
\vdots & \vdots & \ddots & \vdots & \vdots & \vdots & \vdots & \ddots & \vdots & \vdots & \vdots \\
\mathbf{I}_{p} & \mathbf{0} & \cdots & \mathbf{0} & \mathbf{0} & \mathbf{0} & \mathbf{0} & \cdots & \mathbf{I}_{p} & \mathbf{0} & \mathbf{0} \\
\mathbf{A}_{1} & \mathbf{A}_{2} & \cdots & \mathbf{A}_{n-1} & \mathbf{A}_{n} & -\mathbf{B}_{2} & -\mathbf{B}_{3} & \cdots & -\mathbf{B}_{m-1} & -\mathbf{B}_{m} & \mathbf{I}_{p}
\end{array}\right] \\
& \mathbf{G}=\left[\begin{array}{lllllllllll}
\mathbf{B}_{1} & \mathbf{0} & \mathbf{0} & \cdots & \mathbf{0} & \mathbf{I}_{p} & \mathbf{0} & \mathbf{0} & \cdots & \mathbf{0} & -\mathbf{B}_{1}
\end{array}\right]^{T} \\
& \mathbf{D}=\left[\begin{array}{lllllllllll}
\mathbf{0} & \mathbf{0} & \mathbf{0} & \cdots & \mathbf{0} & \mathbf{0} & \mathbf{0} & \mathbf{0} & \cdots & \mathbf{0} & \mathbf{I}_{p}
\end{array}\right]^{T} \\
& \mathbf{H}=\left[\begin{array}{lllllllllll}
\mathbf{I}_{p} & \mathbf{0} & \mathbf{0} & \cdots & \mathbf{0} & \mathbf{0} & \mathbf{0} & \mathbf{0} & \cdots & \mathbf{0} & \mathbf{0}
\end{array}\right]^{T}
\end{aligned}
$$

The state variable feedback control law takes the usual form,

$$
\mathbf{u}(k)=-\mathbf{K} \mathbf{x}(k)
$$

where $\mathbf{K}$ is the PIP control gain matrix. 


\subsection{Control structure}

The final control system can be structurally related to more conventional designs, such as multivariable PI/PID control, as illustrated in Fig. 1. Here, the control gains are represented by,

$$
\begin{gathered}
\mathbf{L}\left(z^{-1}\right)=\mathbf{L}_{0}+\mathbf{L}_{1} z^{-1}+\ldots+\mathbf{L}_{n-1} z^{-n+1} \\
\mathbf{M}\left(z^{-1}\right)=\mathbf{M}_{1} z^{-1}+\ldots+\mathbf{M}_{m-1} z^{-m+1} \\
\mathbf{I}=\mathbf{k}_{I} /\left(1-z^{-1}\right)
\end{gathered}
$$

while $\mathbf{S}$ and $\mathbf{N}$ represent the nonlinear gasifier simulation and the linear NMSS model respectively. Finally, $z^{-1}$ is the backward shift operator.

Note that Fig. 1 illustrates both the forward path structure and the more conventional feedback form of PIP control. The former utilises output from the nonlinear simulation $\mathbf{S}$ to provide the necessary integral action, with the reduced order linear model $\mathbf{N}$ generating an appropriate signal for the $\mathbf{L}\left(z^{-1}\right)$ controller dynamics. By contrast, the feedback structure only utilises the nonlinear output (in practice, the plant output).

Such control structures have important consequences, both for the robustness of the final design to parametric uncertainty, and for the disturbance rejection characteristics. In particular, while the feedback form is generally more robust to uncertainty in the estimated system dynamics, the unity feedback aspect of the forward path form offers disturbance rejection char- 
acteristics that are usually superior, since they are similar in dynamic terms to those associated with the designed command response [13].

However, preliminary closed-loop experiments with the nonlinear gasifier, suggest that both forms of PIP control are sufficiently robust to changes in the load operating condition and coal quality. Rather, it is the disturbance response that requires most attention in order to meet the specifications. For these reasons, the forward path structure is employed for all the results discussed below. Note that, whichever PIP structure is chosen, it is always converted into the equivalent incremental feedback or incremental forward path form of the algorithm [3]. This provides an inherent means of avoiding integral windup when the controller is subjected to constraints on the actuator signal, as in the present example.

\subsection{Control tuning}

The feedback gain matrix $\mathbf{K}$ that minimises the Linear Quadratic (LQ) cost function below, as determined by the steady state solution of the ubiquitous discrete-time matrix Riccati equation [e.g. 14], is utilised for all the results in this paper.

$$
J=\sum_{i=0}^{\infty}\{\mathbf{x}(i) \mathbf{Q} \mathbf{x}(i)+\mathbf{u}(i) \mathbf{R u}(i)\}
$$


Here $\mathbf{Q}$ and $\mathbf{R}$ are symmetric positive semi-definite and symmetric positive definite weighting matrices, respectively.

It is worth noting that, due to the special structure of the non-minimal state vector, the elements of $\{\mathbf{Q}, \mathbf{R}\}$ have particularly simple interpretation, since the diagonal terms directly define weights assigned to the measured variables and integral-of-error states: see equation (3). This contrasts with minimal state space models that represent the same system in a less intuitive manner, requiring each state to be formed from various, often rather abstract, combinations of the input and output signals. In this manner, the non-minimal formulation provides more design freedom than the equivalent minimal case, as discussed by [2].

As will become apparent below, good control of the benchmark system can be achieved by straightforward adjustment of the diagonal elements of $\mathbf{Q}$ and $\mathbf{R}$, with zero off-diagonal elements. In this regard, the notation described in more detail by a number of earlier papers on multivariable PIP control will again be utilised: see e.g. [6]. Here, only the total weightings assigned to (all the present and past values of) each input and output variable, denoted $y_{1}^{w} \ldots y_{4}^{w}$ and $u_{1}^{w} \ldots u_{4}^{w}$ respectively, together with the integral-of-error state weightings, $z_{1}^{w} \ldots z_{4}^{w}$, are selected by the designer. For example, selecting $y_{1}^{w}=10$ implies a total weighting of 10 on CVGAS and all it's past sampled values. In the default case, each of these parameters is set to unity. 


\section{System Identification}

The identification of an appropriate linear control model plays an essential role in meeting the gasifier design specifications. Of particular importance is the model structure chosen; the load operating condition and coal quality disturbance for which it is obtained; and the nature of the input excitation utilised to generate the necessary time series data. The main difficulty encountered is that, while the long term gasifier dynamics dominate the open loop step response, it is the rapid response modes that are of most importance to the specified control objectives.

The choice of sampling rate is, therefore, very important. In fact, open loop experiments indicate that, while the PGAS variable exceeds its allowed limit of $10 \mathrm{kN} / \mathrm{m}^{2}$ within 2 seconds of the specified step disturbance, the new steady state conditions are not reached for approximately 24 hours. Nonetheless, a sampling rate of $0.25 \mathrm{~s}$ is utilised for all the results below, since this offers an adequate description of the short term dynamics and ensures a rapid response to the disturbances.

The research utilises the Simplified Refined Instrumental Variable (SRIV)

algorithm $[9,10]$ to estimate multi-input, single output (MISO) linear Transfer Function (TF) models. For each output variable: $y_{1}(k)$ (CVGAS), $y_{2}(k)$ (MASS), $y_{3}(k)$ (PGAS) and $y_{4}(k)$ (TGAS), these take the form, 


$$
y_{i}(k)=\frac{B_{i, 1}\left(z^{-1}\right)}{A_{i}\left(z^{-1}\right)} u_{1}(k)+\cdots+\frac{B_{i, 4}\left(z^{-1}\right)}{A_{i}\left(z^{-1}\right)} u_{4}(k) \quad(i=1, \ldots, 4)
$$

Here $y_{i}(k)$ and $u_{i}(k)$ are the output and input variables respectively, while $A_{i}\left(z^{-1}\right)$ and $B_{i, j}\left(z^{-1}\right)$ are appropriately defined polynomials in the backward shift operator: see Tables 1 and $2(i, j=1, \ldots, 4)$. Note that it is a straightforward step to convert such TF models into the Matrix Fraction Description given by equation (1).

Finally, for a given physical system, an appropriate model structure first needs to be identified, i.e. the order of the various polynomials. The two main statistical measures employed to help determine these values are the coefficient of determination $R_{T}^{2}$, based on the response error, which is a simple measure of model fit; and the more sophisticated Young Identification Criterion (YIC), which provides a combined measure of fit and parametric efficiency, with large negative values indicating a model which explains the output data well, without over-parameterisation [10].

\subsection{Benchmark Challenge}

Conceptually, the Benchmark Challenge offers three broad options for system identification: 
1. Treat the nonlinear gasifier model as a previously developed and validated simulation as, of course, it really is. For NMSS/PIP design, equation (1) is then obtained from a data-based combined model reduction and linearisation exercise, conducted on the high order nonlinear simulation model. By contrast, other approaches may directly utilise the known equations of the simulation for analytical linearisation.

2. For the purposes of the benchmark challenge, treat the nonlinear model as a surrogate for the real plant. In fact, this is the approach suggested by the organiser [7]. Analytical linearisation is not possible. Furthermore, any open loop experiments for the identification of an appropriate control model, should be conducted by choosing realistic input signals that would not, in practice, damage the system. In particular, it is clear that the bedmass variable should be regulated during such an experiment, to ensure stability.

3. As for option 2 above, but assume that the plant is not available for planned experiments, i.e. time series data should be collected during the normal operation of the plant. An existing control system, such as the multiple-loop PI algorithm suggested [7], should be utilised to regulate all four output variables. 
The three cases above require notably different approaches to system identification. For option 1, the underlying dynamics are best identified by temporarily removing the complication of actuator constraints. Furthermore, since this is a deterministic simulation, a small amplitude pulse signal can be successfully utilised to estimate linear TF models. Such an approach ensures that the benchmark system responds with small perturbations close to the specified operating point, without activating the nonlinearities.

By contrast, in the case of options 2 or 3 , it is clear that the input constraints must remain in place. The specified rate limits for the gasifier system would then preclude use of pulse signals. Furthermore, in these cases, it would be more realistic to include a stochastic measurement noise component, so as to prevent use of unrealistically small input variations. Here, the plant should be sufficiently excited to overcome the measurement noise signal and so reveal the underlying linear dynamics (for a specified operating condition), whilst still minimising any nonlinear distortions: see e.g. [15].

However, using SRIV methods, simulation experiments quickly reveal that all three options yield satisfactory models appropriate for PIP control system design, testifying to the robustness of the approach. For example, Section 3.2 below considers the case when the deterministic simulation is perturbed by a sequence of steps of varying magnitudes. 


\section{$3.2 \quad$ Linear models}

To demonstrate the modelling methodology, the gasifier simulation is perturbed by the input signals shown in Fig. 2, with the corresponding output response illustrated in Fig. 3. Here, each input variable takes a form similar to a Pseudo Random Binary Signal (PRBS) but where the levels are chosen randomly from a Gaussian distribution centred about the steady state 100\% load operating point. These signals are then modified by the Benchmark rate and level constraints.

In this case, the SRIV algorithm, coupled with the YIC and $R_{T}^{2}$ identification criteria, suggest that the gasifier is well represented by four 3rd order MISO models. These typically yield $R_{T}^{2}>0.99$, i.e. over $99 \%$ of the nonlinear simulation response is explained by the linear models. In fact, the linear response also plotted in Fig. 3 (thin trace), is almost indistinguishable from that of the nonlinear simulation (points).

One particular input realisation yields the models given by Tables 1 and 2 . Relatively large input variations are utilised here since these are found to yield low order transfer function models and good closed-loop performance. However, the final control design is not particularly sensitive to this choice of input signal, nor the magnitude of the step sizes, as long as SRIV methods are used, the control weights are re-tuned using the parameter estimates actually 
obtained, and the load operating condition is approximately maintained for the duration of the open-loop experiment (as is the case here: see Fig. 4).

\section{Performance Tests}

The present section of the paper considers the standard performance tests specified by $[7,8]$. Consider in the first instance, a PIP controller based on TF models obtained from open-loop experiments at the $100 \%$ load operating condition. By first converting the TF models given by Tables 1 and 2 into MFD form (1), it is a straightforward exercise to develop the equivalent 24th order linear NMSS representation (2). Solution of the LQ cost function (8), subsequently yields a fixed gain PIP control agorithm (6), suitable for implementation in the forward path form of Fig. 1.

Closed-loop experiments quickly reveal that WCOL is the most problematic input variable for hitting the constraints. For this reason, the associated LQ weighting is selected as $u_{3}^{w}=100$, with all the remaining parameters set to the default unity. This yields a PIP algorithm with an identical structure and similar gains to that previously obtained for the linear benchmark system [6]. Nonetheless, when now applied to the full nonlinear simulation, with appropriate input constraints, the results are either the same or improved compared to those obtained before, as discussed below. 


\subsection{PIP control optimised for $100 \%$ load}

Tables 3 and 4 summarise the results. Here, the Integral of Absolute Error (IAE) is determined at the three standard operating conditions, i.e. 100\% (as designed for), $50 \%$ and $0 \%$ load. Equivalent values for the multiple-loop PI algorithm [7] are given in parenthesis. To illustrate these results, Fig. 5 shows the response to a sine wave disturbance at $50 \%$ load, while the equivalent step disturbance response is shown in Fig. 6. In these figures, the steady state levels have been subtracted from the output variables.

All of the performance requirements at the $100 \%$ and $50 \%$ load operating conditions, for both step and sine wave disturbances, are comfortably met by the PIP algorithm with, in most cases, improved tracking of the set point compared to the multiple-loop PI algorithm supplied by [7]. Even at 0\% load, the only limitation of the PIP design is that the PGAS variable exceeds its allowed limit by 0.03 bar during the sine wave disturbance test. However, in absolute terms, this is less than $2 \%$ of the 11.5 bar set point and even this problem is straightforwardly solved in Section 4.2 below.

Furthermore, the 50-100\% ramp test illustrated in Fig. 7, shows a smooth transition between these operating levels. In this case, compared to the multi-loop PI algorithm, PIP provides considerably improved control of the bedmass variable, at the expense of a slower temperature response. 
Of course, if this latter result proves unsatisfactory in practice, then the TGAS variable may be penalised in the cost function relative to the other variables, as discussed below. In this regard, it should be stressed that control of the load condition was not considered a design objective in this example, although the latter variable is still graphed against its demanded level in Fig. 7. Again, if indirect regulation of the load condition is later included in the design specifications, this can be straightforwardly achieved by further adjustment of the LQ weights.

Finally, the PIP algorithm proves robust to coal quality disturbances, represented by percentage changes from the norm. In particular, none of the output limits are exceeded for the step and sine wave disturbances when the coal quality is ramped up to +8 or -7 at $100 \%$ load, or for even higher magnitudes at $50 \%$ and $0 \%$ load. In fact, Fig. 5 illustrates the response when the coal quality is ramped down to $-10 \%$, since these results are similar to the case without such a disturbance. For larger coal quality variations, the temperature variable sometimes exceeds its limit, although closed-loop stability is maintained at all times.

Note that, when analysing the response to a coal disturbance, the simulation is always solved for longer than the 300 seconds specified by the standard tests. This is because the input variables often hit level constraints during coal disturbances, which can result in an eventual drift of the outputs. 


\subsection{LQ weighting matrices}

Because of the special structure of the NMSS model, the LQ weightings can be straightforwardly adjusted in order to meet other performance requirements. For example, by increasing the error weighting on the TGAS variable, tracking of temperature and load in the ramp test is improved in comparison to Fig. 7, at the expense of the other variables.

Similar trial and error adjustment of the weighting terms so that $u_{1}^{w}=50$, $u_{2}^{w}=25, u_{3}^{w}=100$ and $u_{4}^{w}=25$, yields a PIP algorithm that successfully maintains the PGAS variable within the limits, even for the problematic $0 \%$ load sine wave disturbance response, as illustrated by Fig. 8. For reference, the equivalent step disturbance response is illustrated in Fig. 9. This latter PIP algorithm meets all the design specifications, although the overall performance is arguably not as good as the earlier PIP design.

In particular, with these modified weights, some of the performance specifications (such as the maximum disturbance off-set or the IAE) are improved in comparison to the initial design above, whilst others are poorer. The exact differences between these two PIP designs varies for each disturbance type and operating level; hence, for brevity, the details are omitted here.

It is clear that any further fine tuning of the algorithm depends on more detailed design specifications. In this regard, one technique for automatically 
mapping such objectives into elements of the weighting matrices, is multiobjective optimisation in its goal attainment form [12]. Here, the designer would benefit from knowledge of the relative importance of each output variable; and whether it is the peak value, or the long term integral of absolute error, of a given variable that has the most critical effect on the gasifier performance.

The latter comment is particularly true of the $50-100 \%$ ramp test, where Fig. 7 represents just one particular PIP realisation, not necessarily the optimal response in terms of the gasifier system. It is noteworthy, for example, that the temperature constraint is $\pm 1 \mathrm{~K}$, compared to a set point of over $1000 \mathrm{~K}$. If this proves to be a genuine requirement, then the LQ weightings may be modified appropriately.

\subsection{PIP control optimised for $50 \%$ load}

The linear models in the discussion above are all based on data collected at the $100 \%$ load operating condition, since this is the normal operating state of the plant. However, since the performance tests cover the full range 0-100\%, there is an argument for designing the controller at $50 \%$ instead, i.e. in the middle of the operating range. In this case, the TF models take a similar structure those in Tables 1 and 2, although clearly the parameters 
and hence control algorithm differ. Here, PIP control performance at the lower operating conditions are improved, at the expense of a small reduction in the performance at $100 \%$ load, as would be expected.

Since the latter performance degradation is minimal, these results potentially suggest that utilisation of the $50 \%$ operating condition for the design of a fixed gain PIP controller is the preferred option. However, this conclusion requires a more detailed consideration of the control objectives than provided for the purposes of the present challenge. For example, what percentage of time is the actual system close to the $100 \%$ load condition? Again, however, the flexibility of the NMSS/PIP approach emerges - changing the optimal operating condition of the controller requires data collection from just one open loop experiment similar to Fig. 3, followed by minimal tuning of the LQ weights.

\subsection{Modified input constraints}

With regards to the coal quality disturbance, simulation trials suggest that the WCOL and WCHR variables are particularly important. These represent the coal input and char extraction flow rates respectively. For example, a high quality coal input (disturbance $+18 \%$ ) naturally requires less char extraction because it would be expected to have a low residual, hence WCOL and 
WCHR will inevitably approach the level constraints.

In this regard, whilst it is interesting to use advanced control theory in an attempt to solve the benchmark specifications, from a practical point of view, the gasifier problem becomes rather straightforward if the WCHR and WCOL constraints are softened somewhat. Clearly the latter option represents one particular (hardware) solution to this control problem.

\section{Conclusions}

This paper has discussed the application of Proportional-Integral-Plus (PIP) control methods to the ALSTOM Benchmark Challenge II. The approach is based on the identification of discrete-time transfer function models using the Simplified Refined Instrumental Variable (SRIV) algorithm. Here, a very straightforward design process is employed, requiring one open loop experiment and automatic selection of a linear model. Adequate closed loop PIP control responses are then obtained by manually tuning the intuitive weighting parameters.

The design effort took less than 5 hours, although clearly the authors are very familiar with the approach, have ready access to the necessary software tools and had previously studied the linear challenge. Note also, that the PIP controller considered here has a similar implementational complexity 
to conventional PI/PID designs, requiring only the addition of a multivariable structure and storage of additional past values. Of course, since this is a discrete-time algorithm, these requirements are very straightforward to program for a digital PC.

However, the PIP algorithm may be extended in various ways, albeit at the cost of increasing complexity. For example, while the basic form utilises an incremental structure to account for the input constraints, such an ad hoc approach does not necessarily yield optimal control performance in other applications. Although beyond the scope of the present paper, one research area currently being investigated in this regard, exploits the advantages of the NMSS representation within a conventional model-predictive control form.

Finally, one limitation of the discrete-time PIP algorithm, is that it takes up to 1 sampling interval before the controller starts to respond to a disturbance input. In simulation, this puts the approach at a disadvantage against continuous-time designs such as [7]. In this context, it should also be pointed out that all the SRIV/PIP methods discussed in the present paper, are readily developed in continuous time, providing another avenue for further research and potentially improved results.

Nonetheless, the discrete-time, linear PIP algorithm considered here, successfully satisfies all the control specifications for all three operating conditions, even with significant coal disturbances. 


\section{Acknowledgments}

The authors are grateful for the support of the Engineering and Physical Sciences Research Council (EPSRC). The system identification tools utilised in this research have been assembled as the Captain toolbox within the Matlabß software environment [16].

\section{References}

[1] P. C. Young, M. A. Behzadi, C. L. Wang, and A. Chotai. Direct digital and adaptive control by input-output, state variable feedback pole assignment. International Journal of Control, 46:1867-1881, 1987.

[2] C. J. Taylor, P. C. Young, and A. Chotai. State space control system design based on non-minimal state-variable feedback : Further generalisation and unification results. International Journal of Control, 73:13291345,2000 .

[3] C. J. Taylor, P. C. Young, A. Chotai, and J. Whittaker. Non-minimal state space approach to multivariable ramp metering control of motorway bottlenecks. Proceedings of the Institution of Electrical Engineers, Control Theory and Applications, 145(6):568-574, 1998.

[4] C. J. Taylor, P. A. Leigh, A. Chotai, P. C. Young, E. Vranken, and 
D. Berckmans. Cost effective combined axial fan and throttling valve control of ventilation rate. Proceedings of the Institution of Electrical Engineers, Control Theory and Applications, 151(5):577-584, 2004.

[5] J. Gu, C. J. Taylor, and D. W. Seward. The automation of bucket position for the intelligent excavator lucie using the proportional-integralplus (pip) control strategy. Journal of Computer-Aided Civil and Infrastructure Engineering, 12:16-27, 2004.

[6] C. J. Taylor, A. P. McCabe, P. C. Young, and A. Chotai. Proportionalintegral-plus (pip) control of the alstom gasifier problem. Proceedings of the Institution of Mechanical Engineers, Journal of Systems and Control Engineering, 214:469-480, 2000.

[7] R. Dixon. Alstom benchmark challenge 2: Control of a non-linear gasifier model. ALSTOM Power Technology Centre, Whetstone, Leicester, UK, 2002 .

[8] R. Dixon and A. W. Pike. Alstom benchmark challenge on gasifier control. Proceedings of the Institution of Electrical Engineers, Control Theory and Applications, 2005.

[9] P. C. Young. Recursive Estimation and Time Series Analysis. SpringerVerlag, Berlin, 1984. 
[10] P. C. Young. Simplified refined instrumental variable (sriv) estimation and true digital control (tdc): a tutorial introduction. In 1st European Control Conference, pages 1295-1306, Grenoble, 1991.

[11] P. C. Young, M. Lees, A. Chotai, W. Tych, and Z. S. Chalabi. Modelling and pip control of a glasshouse micro-climate. Control Engineering Practice, 2(4):591-604, 1994.

[12] A. Chotai, P. C. Young, P. McKenna, and W. Tych. Pip design for delta-operator systems: Part 2, mimo systems. International Journal of Control, 70:149-168, 1998.

[13] C. J. Taylor, A. Chotai, and P. C. Young. Proportional-integral-plus (pip) control of time delay systems. Proceedings of the Institution of Mechanical Engineers, Journal of Systems and Control Engineering, 212:37-48, 1998.

[14] J.A. Borrie. Modern Control Systems: A Manual of Design Methods. Prentice-Hall, 1986.

[15] K.R. Godfrey, A.H. Tan, and H.A. Barker. A survey of readily accessible perturbation signals. In 13th IFAC Symposium on System Identification, pages 653-658, Rotterdam, August 2003.

[16] P. C. Young, C. J. Taylor, D. J. Pedregal, W. Tych, and P. G. McKenna. 
Captain Toolbox for time series analysis and forecasting. Centre for Research on Environmental Systems and Statistics, Lancaster University, www.es.lancs.ac.uk/cres/captain, 2004. 
Table 1: Denominator polynomials.

\begin{tabular}{cc} 
Output $i$ & $A_{i}\left(z^{-1}\right)$ \\
\hline CVGAS & $A_{1}\left(z^{-1}\right)=1-2.9502 z^{-1}+2.9009 z^{-2}-0.9507 z^{-3}$ \\
MASS & $A_{2}\left(z^{-1}\right)=1-2.7001 z^{-1}+2.4169 z^{-2}-0.7168 z^{-3}$ \\
PGAS & $A_{3}\left(z^{-1}\right)=1-1.7028 z^{-1}+0.5964 z^{-2}+0.1133 z^{-3}$ \\
TGAS & $A_{4}\left(z^{-1}\right)=1-1.6440 z^{-1}+0.4625 z^{-2}+0.1815 z^{-3}$ \\
\hline
\end{tabular}


Table 2: Numerator polynomials.

\begin{tabular}{|c|c|c|}
\hline Output $i$ & Input $j$ & $B_{i, j}\left(z^{-1}\right)$ \\
\hline CVGAS & WCHR & $B_{1,1}\left(z^{-1}\right)=0.0017 z^{-1}-0.0017 z^{-2}$ \\
\hline CVGAS & WAIR & $B_{1,2}\left(z^{-1}\right)=-2.2586 z^{-1}+4.4468 z^{-2}-2.1881 z^{-3}$ \\
\hline CVGAS & WCOL & $B_{1,3}\left(z^{-1}\right)=1.5498 z^{-1}-3.0291 z^{-2}+1.4793 z^{-3}$ \\
\hline CVGAS & WSTM & $B_{1,4}\left(z^{-1}\right)=-2.1201 z^{-1}+4.3281 z^{-2}-2.2079 z^{-3}$ \\
\hline MASS & WCHR & $B_{2,1}\left(z^{-1}\right)=-0.0283 z^{-1}-0.0012 z^{-2}+0.0252 z^{-3}$ \\
\hline MASS & WAIR & $B_{2,2}\left(z^{-1}\right)=-0.0053 z^{-1}+0.0033 z^{-2}$ \\
\hline MASS & WCOL & $B_{2,3}\left(z^{-1}\right)=0.0232 z^{-1}+0.0002 z^{-2}-0.0210 z^{-3}$ \\
\hline MASS & WSTM & $B_{2,4}\left(z^{-1}\right)=-0.0006 z^{-1}-0.0014 z^{-2}-0.0011 z^{-3}$ \\
\hline PGAS & WCHR & $B_{3,1}\left(z^{-1}\right)=0.0017 z^{-1}+0.0017 z^{-2}$ \\
\hline PGAS & WAIR & $B_{3,2}\left(z^{-1}\right)=1.0733 z^{-1}-1.1540 z^{-2}+0.1441 z^{-3}$ \\
\hline PGAS & WCOL & $B_{3,3}\left(z^{-1}\right)=0.4770 z^{-1}-0.3654 z^{-2}-0.0772 z^{-3}$ \\
\hline PGAS & WSTM & $B_{3,4}\left(z^{-1}\right)=1.1725 z^{-1}-0.7965 z^{-2}-0.2628 z^{-3}$ \\
\hline TGAS & WCHR & $B_{4,1}\left(z^{-1}\right)=0.0012 z^{-1}+0.0015 z^{-2}$ \\
\hline TGAS & WAIR & $B_{4,2}\left(z^{-1}\right)=0.1562 z^{-1}-0.2828 z^{-2}+0.1286 z^{-3}$ \\
\hline TGAS & WCOL & $B_{4,3}\left(z^{-1}\right)=-0.0183 z^{-1}+0.0159 z^{-2}$ \\
\hline TGAS & WSTM & $B_{4,4}\left(z^{-1}\right)=-0.0743 z^{-1}+0.1256 z^{-2}-0.0517 z^{-3}$ \\
\hline
\end{tabular}


Table 3: IAE for sine wave.

\begin{tabular}{ccccc} 
Load & CVGAS & MASS & PGAS & TGAS \\
\hline $100 \%$ & 0.14 & 0.09 & 5.45 & 49.30 \\
$(\mathrm{PI})$ & $(0.76)$ & $(2.07)$ & $(9.18)$ & $(66.15)$ \\
\hline $50 \%$ & 0.28 & 0.10 & 6.60 & 61.16 \\
$(\mathrm{PI})$ & $(0.87)$ & $(2.52)$ & $(11.45)$ & $(73.80)$ \\
\hline $0 \%$ & 1.07 & 1.33 & 16.84 & 105.59 \\
$(\mathrm{PI})$ & $(1.03)$ & $(3.00)$ & $(18.93)$ & $(79.14)$ \\
\hline
\end{tabular}

Table 4: IAE for step disturbance.

\begin{tabular}{ccccc}
\hline Load & CVGAS & MASS & PGAS & TGAS \\
\hline $100 \%$ & 0.03 & 0.01 & 0.66 & 78.55 \\
$(\mathrm{PI})$ & $(0.03)$ & $(0.80)$ & $(0.39)$ & $(32.52)$ \\
\hline $50 \%$ & 0.03 & 0.04 & 0.30 & 18.95 \\
$(\mathrm{PI})$ & $(0.03)$ & $(0.42)$ & $(0.47)$ & $(38.53)$ \\
\hline $0 \%$ & 0.06 & 0.06 & 0.50 & 49.90 \\
$(\mathrm{PI})$ & $(0.04)$ & $(0.66)$ & $(0.60)$ & $(38.49)$ \\
\hline
\end{tabular}




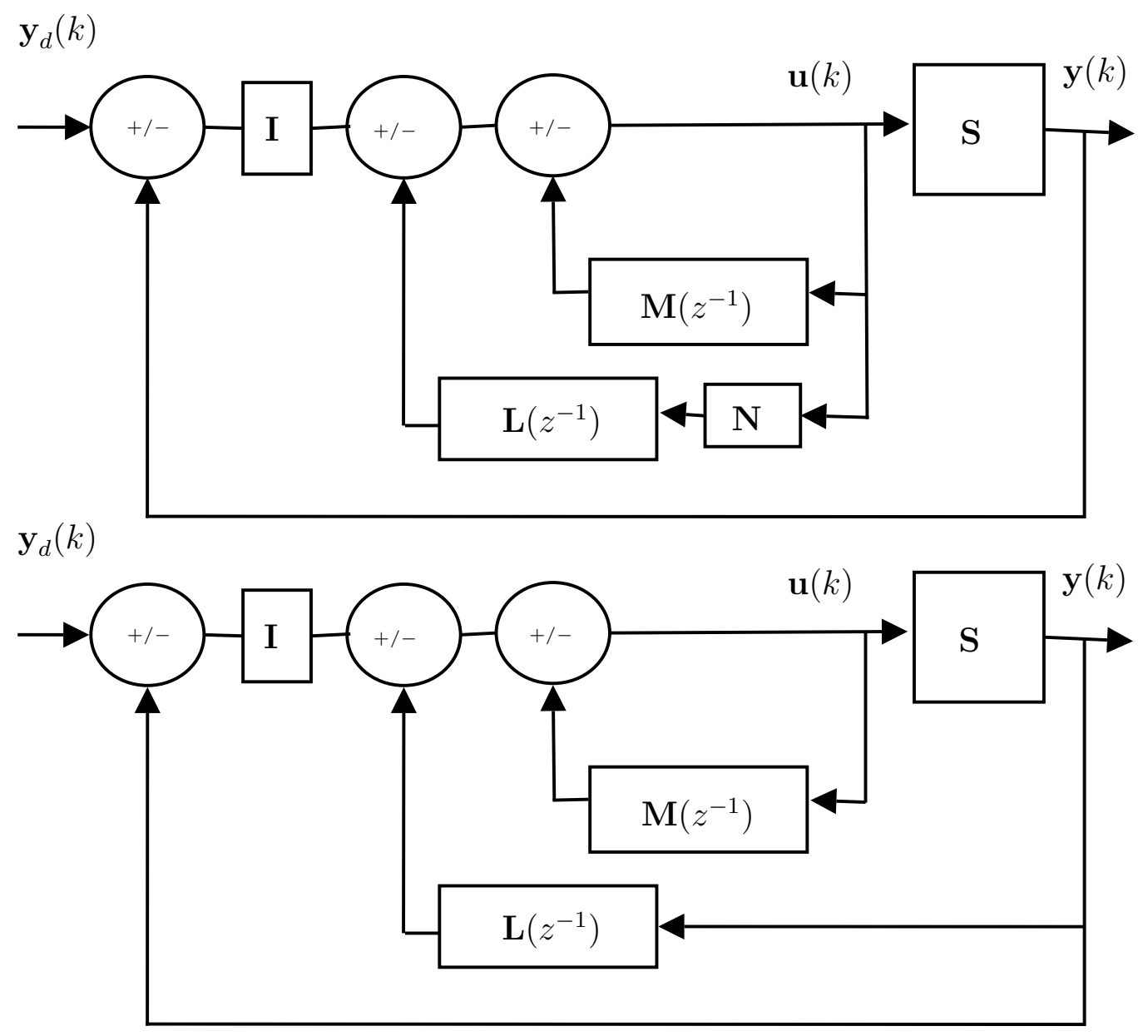

Figure 1: Multivariable PIP control implemented in forward path (top) and feedback (bottom) form. 

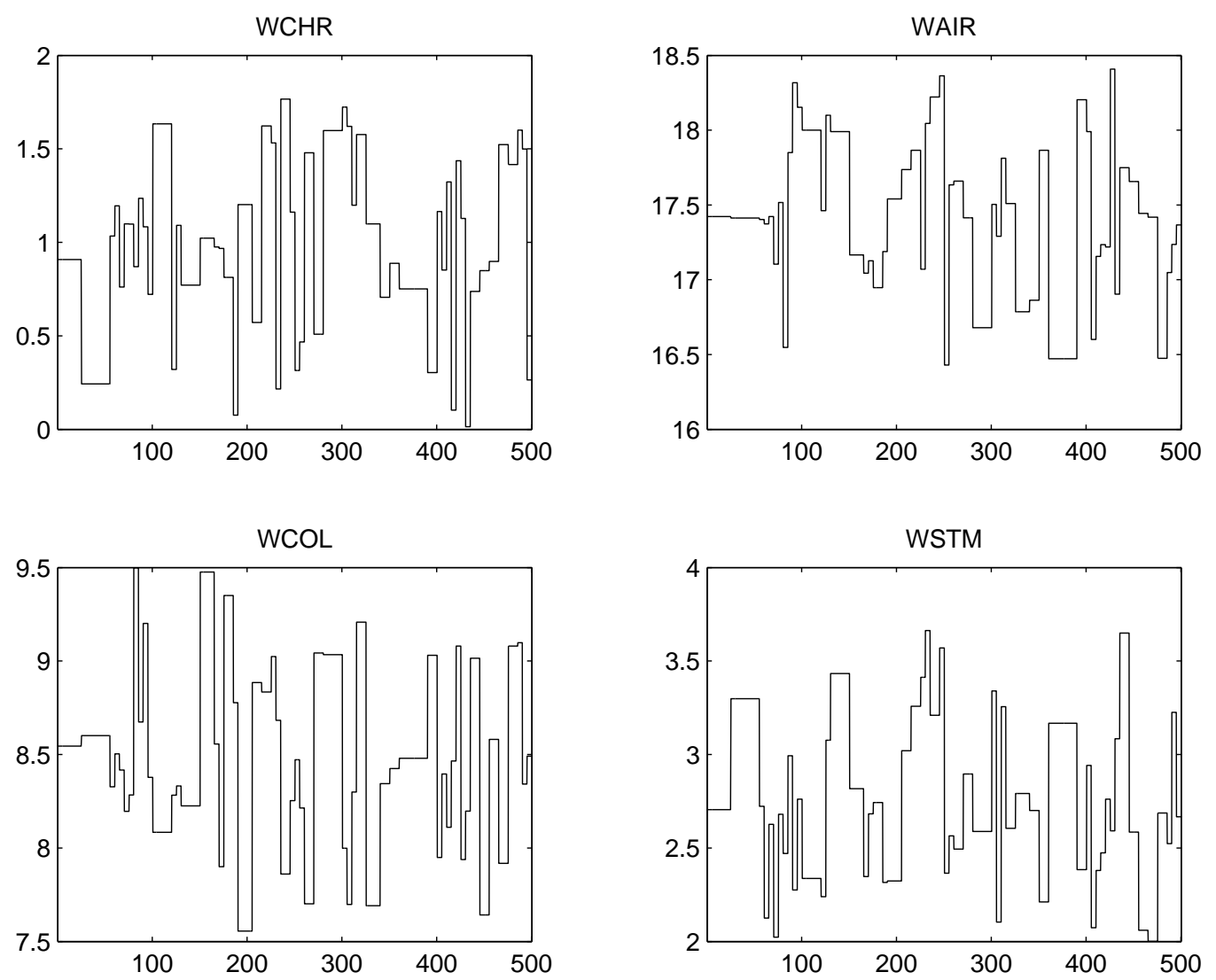

Figure 2: Input variables for open-loop experiment. 

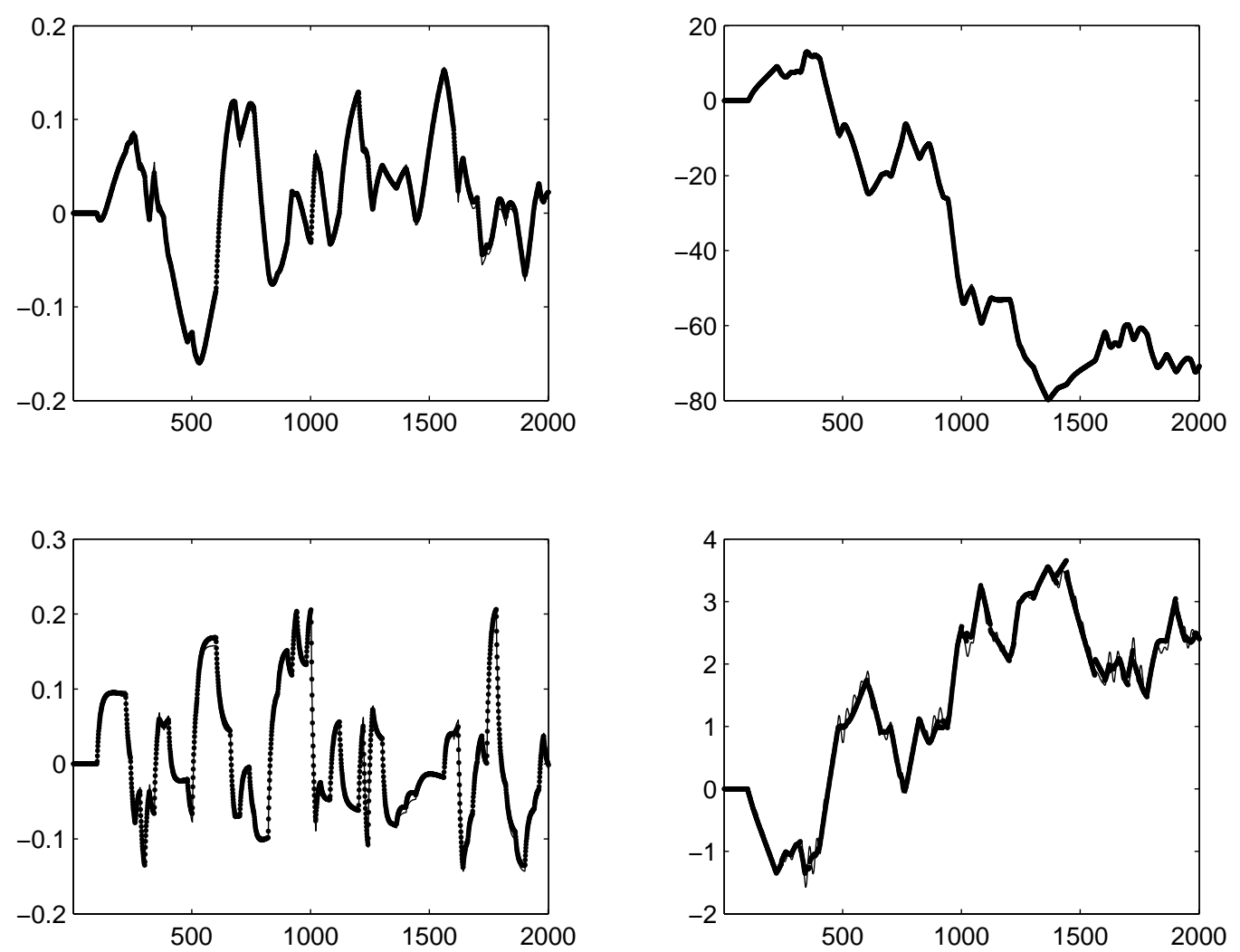

Figure 3: Output variables for open-loop experiment. 


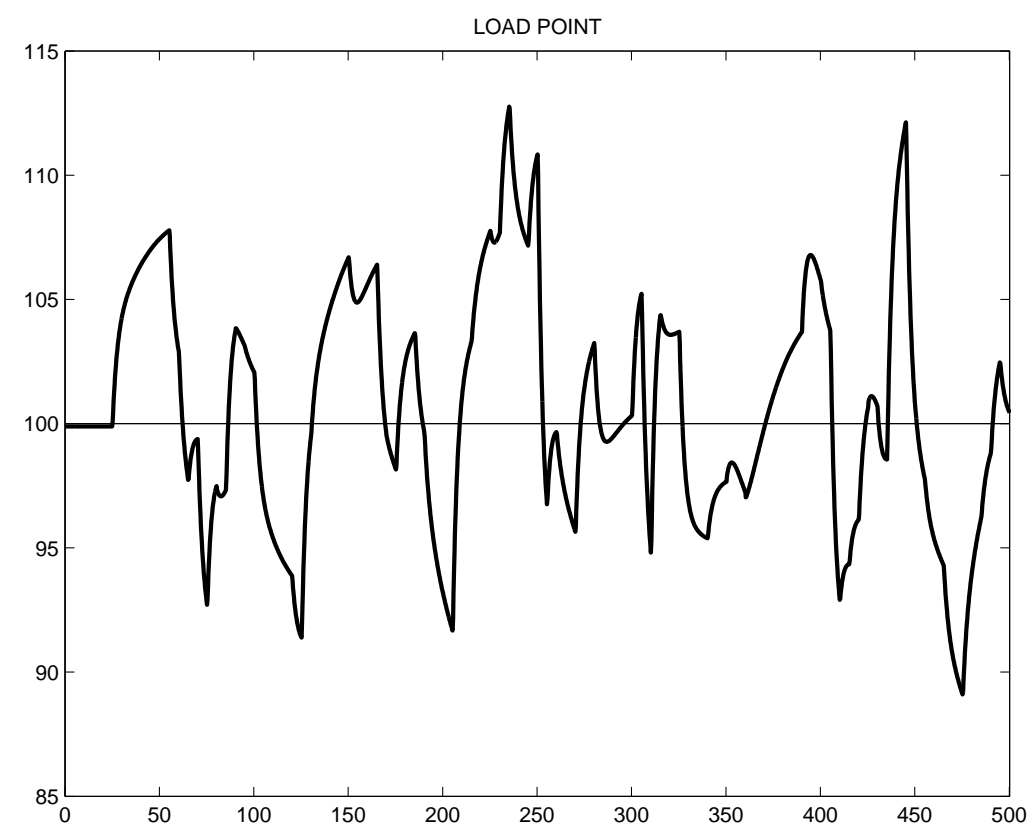

Figure 4: Load for open-loop experiment. 

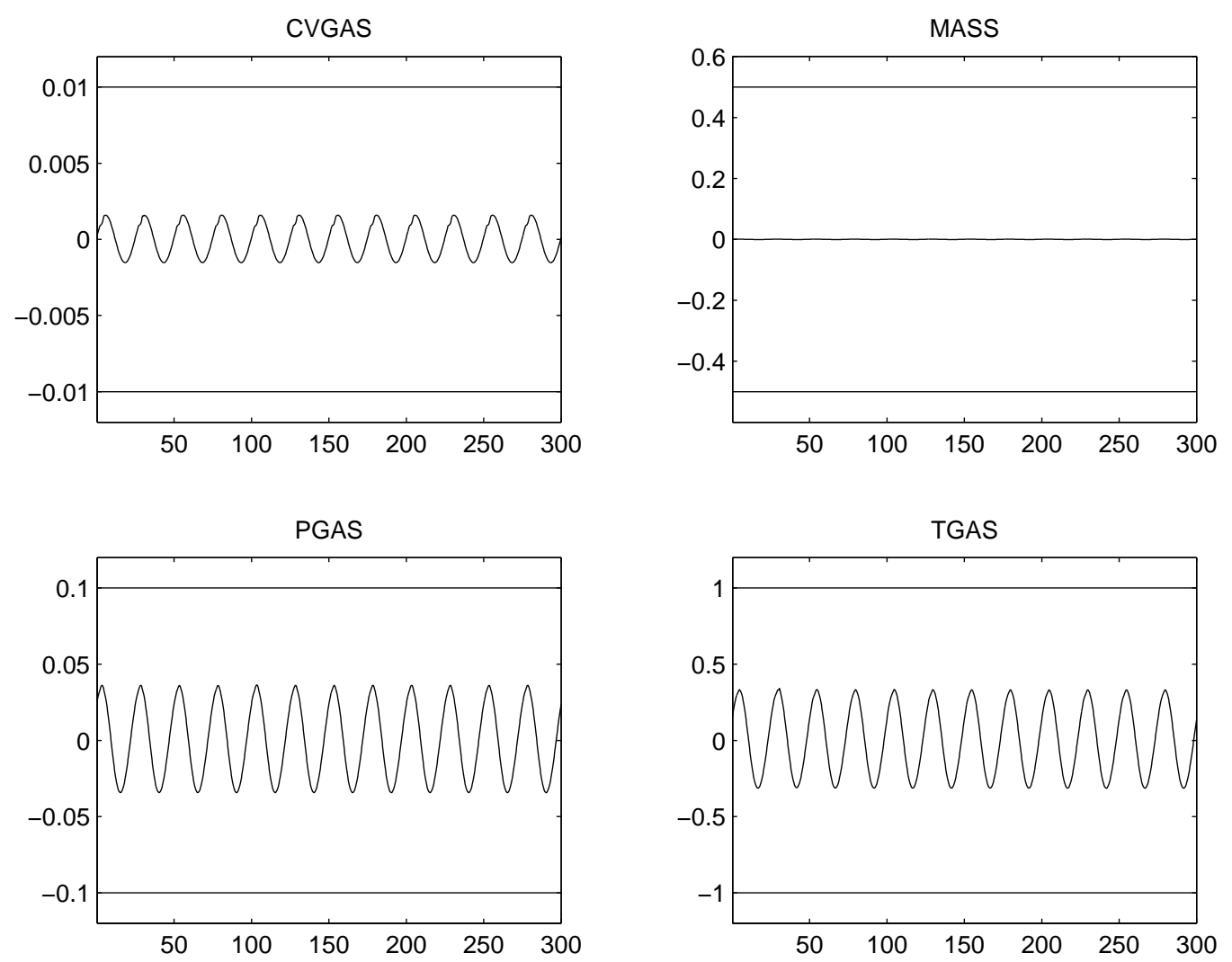

Figure 5: Sine wave disturbance, $50 \%$ load, coal quality variation $-10 \%$. 

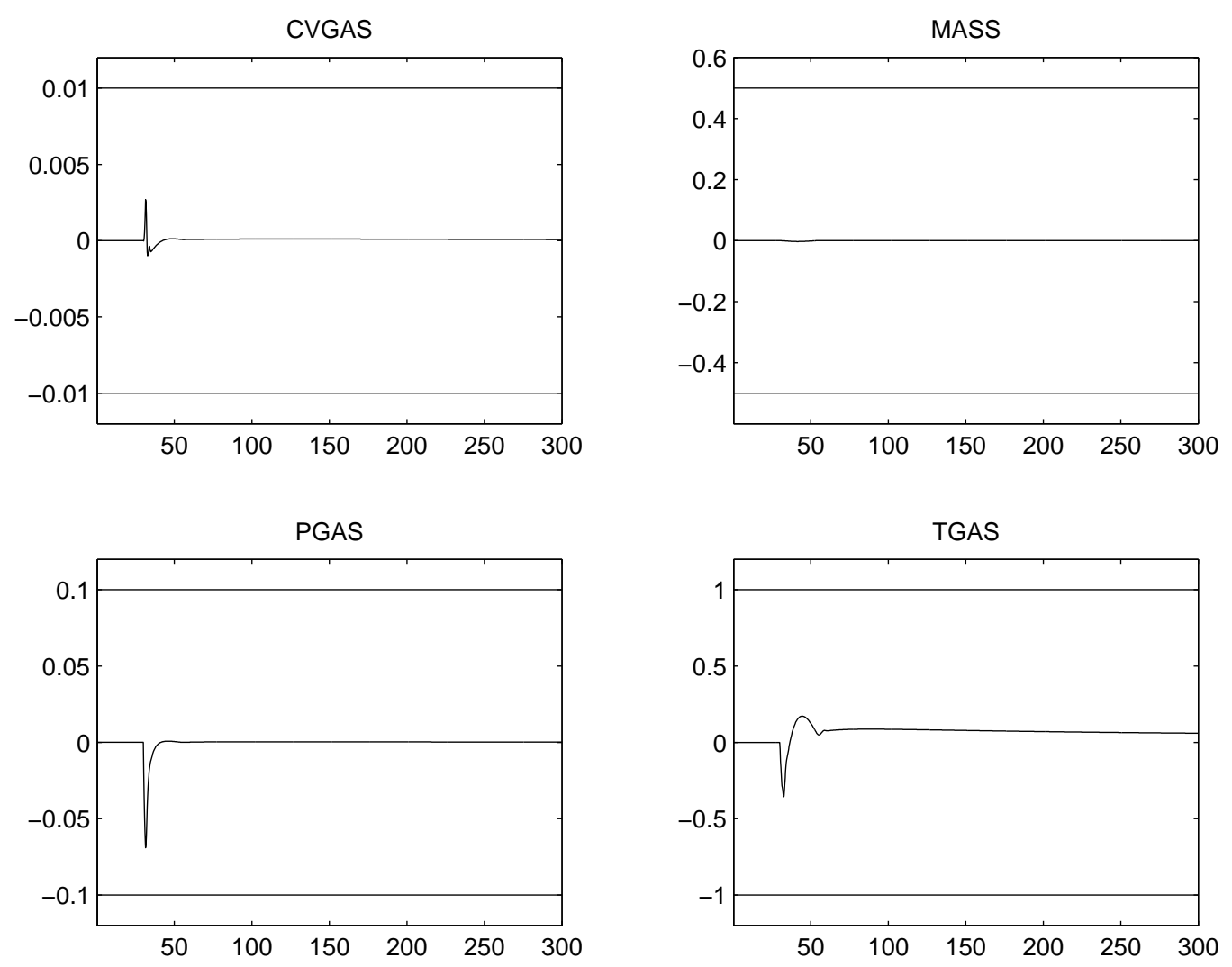

Figure 6: Step disturbance, 50\% load, coal quality variation $0 \%$ 

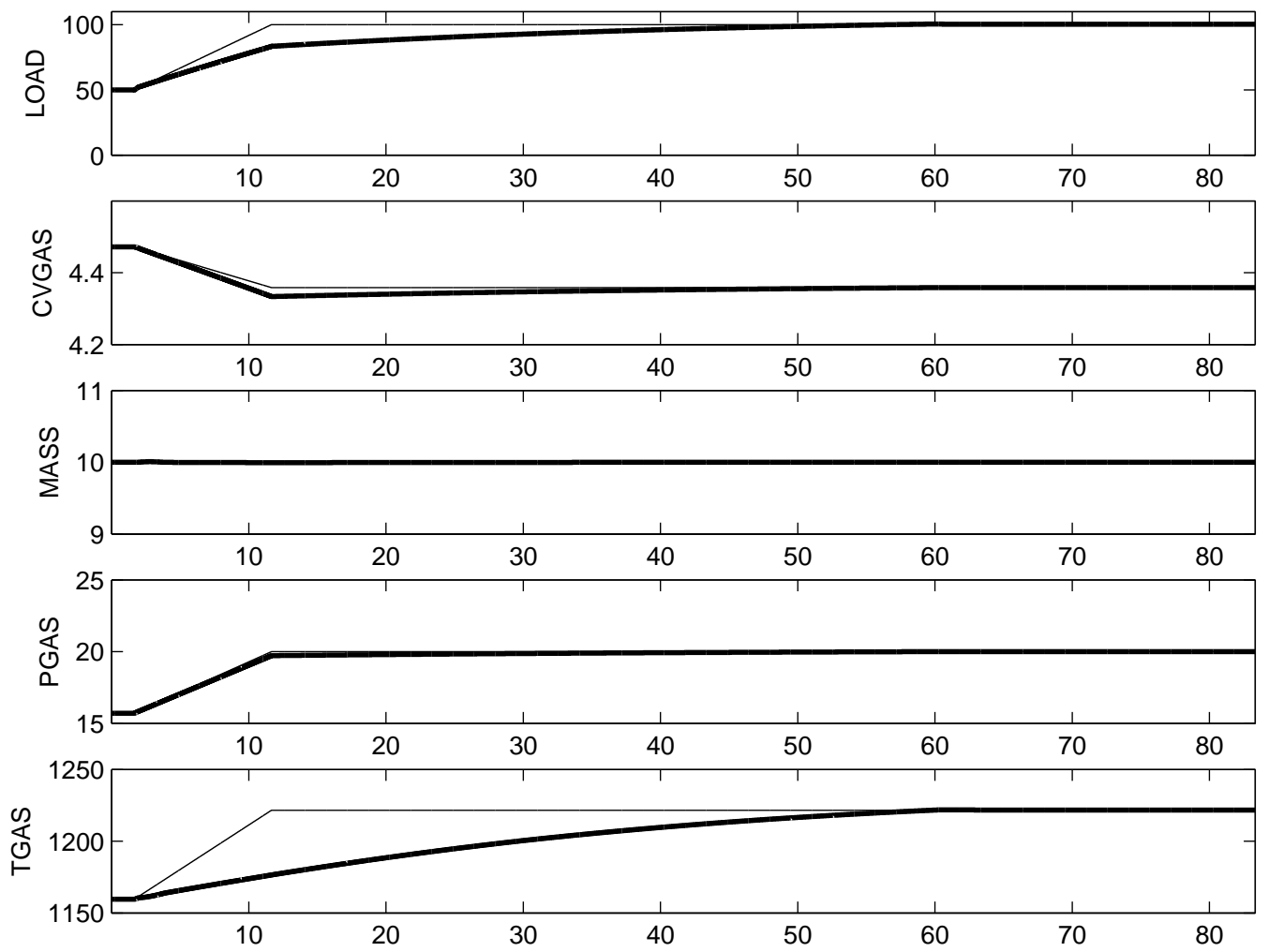

Figure 7: Load 50-100\% ramp test. 

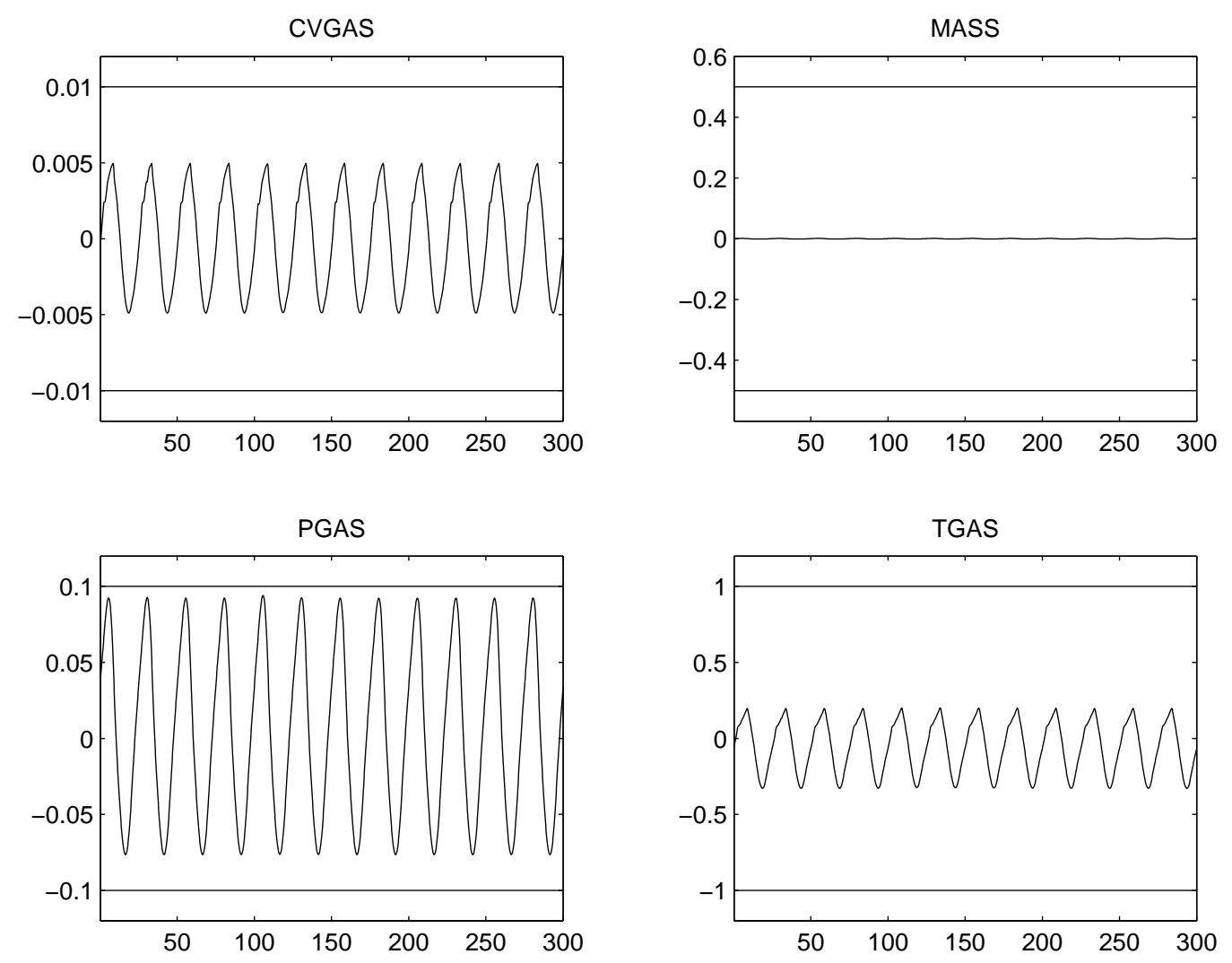

Figure 8: Sine wave, 0\% load, revised LQ weights. 

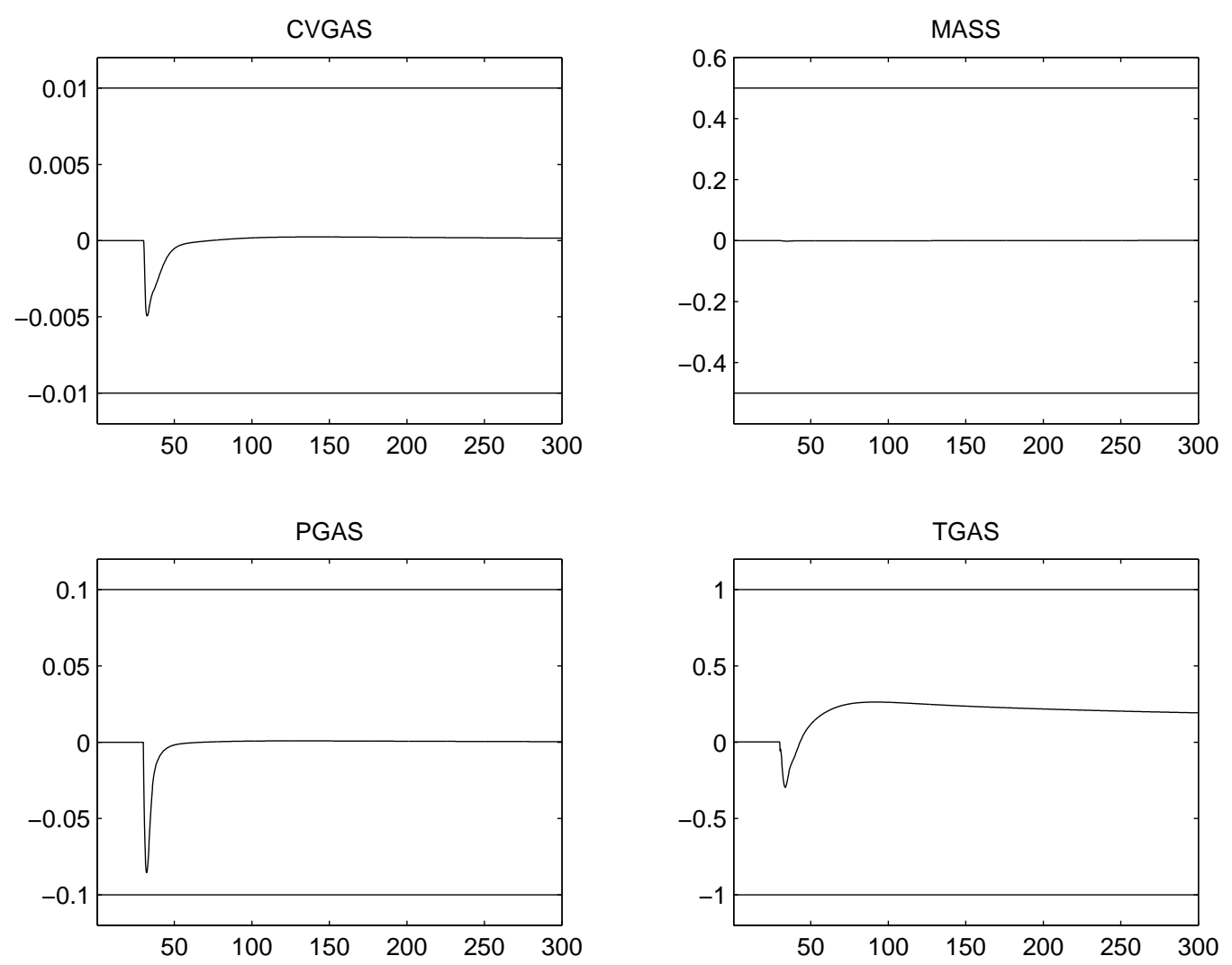

Figure 9: Step disturbance, 0\% load, revised LQ weights. 